\title{
Références bibliographiques du dossier « Les grands débats éducatifs aujourd'hui »
}

\section{Maryannick Vervisch-Lestrade}

\section{(2) OpenEdition}

Édition électronique

URL : http://journals.openedition.org/ries/3187

DOI : 10.4000/ries.3187

ISSN : 2261-4265

\section{Éditeur}

Centre international d'études pédagogiques

\section{Édition imprimée}

Date de publication : 1 septembre 1997

Pagination : 145-151

ISSN : 1254-4590

\section{Référence électronique}

Maryannick Vervisch-Lestrade, «Références bibliographiques du dossier « Les grands débats

éducatifs aujourd'hui » », Revue internationale d'éducation de Sèvres [En ligne], 15 | 1997, mis en ligne le 08 juillet 2013, consulté le 03 mai 2019. URL : http://journals.openedition.org/ries/3187 ; DOI :

$10.4000 /$ ries.3187

Ce document a été généré automatiquement le 3 mai 2019.

(c) Tous droits réservés 


\title{
Références bibliographiques du dossier «Les grands débats éducatifs aujourd'hui »
}

\author{
Maryannick Vervisch-Lestrade
}

1 Cette bibliographie thématique présente une sélection de documents récents, français et étrangers, consultables au CIEP. Liée au dossier de la revue, elle est organisée par rapport à la logique de celui-ci. La plupart des références y sont donc classées selon l'ordre alphabétique des grandes régions du monde, les documents présentant un caractère international figurant en tête de la liste.

\section{International}

BANQUE MONDIALE. Priorités et stratégies pour l'éducation: une étude de la Banque mondiale, Washington: Banque mondiale, 1995, XVI- 195 p. (Le développement à l'œeuvre).

Dans les pays en développement, l'éducation a progressé, mais il est encore nécessaire d'en élargir l'accès dans certains cas, d'en améliorer l'équité et la qualité et, éventuellement, d'en accélérer la réforme. En ce qui concerne le financement, l'éducation de base doit être privilégiée dans les dépenses publiques. II est aussi nécessaire de trouver de nouvelles sources de fonds et de réaliser des gains d'efficacité permettant des économies. Tout cela conduit, dans la perspective d'une participation accrue des familles et d'une autonomie grandissante des établissements, à une redéfinition du rôle de l'État pour faciliter la mise en œuvre du changement.

3 BOULAHIA Souad éd., et al. "Éducation de base et pédagogie de la réussite: promouvoir le changement ", La Revue des échanges, sept. 1995, vol. 12, n 3, p. 1-29.

Depuis la conférence mondiale de Jomtien sur l'éducation pour tous, favoriser l'accès de tous à l'éducation de base et augmenter la pertinence et l'efficacité des apprentissages scolaires sont des préoccupations croissantes. Ce dossier présente, sur ce thème, des 
textes de synthèse et des réflexions de chefs d'établissement et inspecteurs de plusieurs pays : Bénin, Belgique, Canada, France, Suisse, Tunisie.

BUREAU INTERNATIONAL D'ÉDUCATION (Genève). Données mondiales sur l'éducation = Datos mundiales de educacion = World Data on Education : 1993-1994. Genève $:$ BIE, juil. 1996, $1 \mathrm{~cd}$ ROM, $P C$, Windows 3.1, $4 M B R A M+1$ brochure utilisateur.

Sont regroupés sur ce cédérom les rapports faits sur leur système éducatif, par les pays ayant participé à la $44^{\mathrm{e}}$ conférence internationale sur l'éducation, tenue du 3 au 8 octobre 1994 à Genève.

CONFÉRENCE DES MINISTRES DE L'ÉDUCATION DES PAYS AYANT LE FRANÇAIS EN PARTAGE (Dakar). L'éducation de base: vers une nouvelle école: document de réflexion et d'orientation. Dakar: CONFEMEN, 1995, $94 \mathrm{p}$.

Après avoir présenté les causes et les conséquences de la crise des systèmes éducatifs, ce document montre l'importance, pour l'insertion des jeunes, d'une éducation de base adaptée et efficace. Pour la Conférence, la construction d'une "nouvelle école de base " est un projet ambitieux, exigeant et réaliste, de toute façon indispensable.

CYPEL éd., BARBA Sylvie coord., FALGA Pierre coord., et al. "L'école demain». Courrier international, 21-27 sept. 1995, $n^{\circ} 255$, p. I-XXVIII.

Ce supplément au numéro regroupe des articles de journalistes de plusieurs pays sur certains aspects de leur système éducatif. Même si les particularités nationales sont importantes, beaucoup de pays connaissent une crise du système scolaire, qui touche ses différents acteurs : élèves, parents, enseignants. Cependant, les innovations éducatives existent, aussi bien en direction des nouvelles technologies que pour favoriser l'intégration et l'égalité des chances. Le débat sur les évolutions nécessaires pour un enseignement adapté et tourné vers l'avenir reste largement ouvert.

DELORS Jacques coord., COMMISSION INTERNATIONALE SUR L'ÉDUCATION POUR LE XXI ${ }^{e}$ SIECLE rapport. L'éducation : un trésor est caché dedans : rapport à l'Unesco. Paris: Unesco: $O$. Jacob, 1996, $311 p$.

À la demande de l'Unesco, la Commission, regroupant des personnalités venues d'horizons géographiques, culturels et professionnels variés, a réfléchi sur les défis à relever par l'éducation dans les années à venir et préparé suggestions et recommandations. Le rapport issu de ses travaux montre l'importance de l'éducation pour la compréhension du monde, la démocratie et le développement. Il évoque ensuite les objectifs de l'éducation et sa nécessaire continuité au long de la vie. Les orientations proposées concernent les différents niveaux d'enseignement, le rôle des enseignants, les choix politiques et la coopération internationale.

FISKE Edward B. Decentralization of Education: Politics and Consensus. Washington: World Bank, 1996, VIII-39 p., (Directions in development).

Ce document est destiné aux décideurs et praticiens de l'éducation dans les pays en développement, ainsi qu'aux organismes de coopération dans ce domaine. Après une étude de cas sur la Colombie, la brochure présente les dimensions politiques, les raisons et les effets de la décentralisation, en citant l'exemple de différents pays et en terminant sur la nécessaire construction d'un consensus.

Rapport mondial sur l'éducation 1995. Paris: Unesco, 1996, $173 p$.

L'édition 1995 de ce rapport biennal a pour thème central l'éducation des femmes et des filles. Le document examine les tendances globales et les progrès en matière d'accès des femmes à l'enseignement et met l'accent sur les disparités hommes/femmes. Il comporte 
aussi une brève analyse des mesures prises pour l'enseignement de la paix, des droits de l'homme et de la démocratie.

10 TUIJNMAN Albert rap., HASAN Abrar dir. Apprendre à tout âge: réunion du Comité de l'éducation au niveau ministériel, 16-17 janvier 1996. Paris : OCDE, 1996, $363 \mathrm{p}$.

Ce comité s'est réuni sur le thème « Faire de l'apprentissage à vie une réalité pour tous ». L'apprentissage comme processus ininterrompu est jugé nécessaire pour atteindre les objectifs éducatifs, sociaux et économiques fixés. Les stratégies de mise en œuvre seront adaptées au pays concerné; les contextes politique, culturel et l'omniprésence des technologies de l'information et de la communication orienteront les actions. Les priorités concerneront notamment les points suivants: l'éducation préscolaire et l'enseignement obligatoire; l'articulation entre enseignements formels, informels et vie professionnelle; le rôle des pouvoirs publics; les bases de connaissances et de compétences ; le financement de cet apprentissage.

\section{Afrique - Amérique - Asie}

11 AEDO-RICHMOND Ruth ed. "Special Issue on Education in Latin America.» Compare: a Journal of Comparative Education, juin 1996, vol. 26, $n^{\circ} 2, p .131-252$.

Les différents articles portent sur: une comparaison de l'enseignement post-obligatoire aux États- Unis et au Mexique; l'éducation interculturelle au Venezuela; le développement de l'éducation des indigènes au Pérou; les récents changements des programmes dans l'enseignement primaire et la réforme de l'enseignement secondaire au Chili. Une abondante bibliographie sur l'éducation dans les différents pays d'Amérique latine complète le dossier.

BRANDOLIN Joseph. Réinventer l'éducation en Afrique. $2^{e}$ tirage. Montrouge (France): Afrique éducation, 1996, $191 \mathrm{p}$.

Les systèmes éducatifs africains connaissent des difficultés et les modèles importés ne correspondent pas aux besoins. Il faut donc réinventer l'éducation en Afrique aux différents niveaux d'enseignement, en tenant compte des échecs mais aussi des expériences réussies, en s'appropriant les nouvelles technologies et en associant à ce projet toutes les composantes d'un pays, y compris l'important secteur informel. Dans cette optique, c'est en un véritable partenariat de développement que doit se transformer la coopération internationale dans ce secteur.

HENEVELD Ward, CRAIG Helen. Schools Count: World Bank Project Designs and the Quality of Primary Education in Sub-Saharan Africa. Washington: World Bank, 1996, XVIII-127 p. (World Bank technical paper, 303).

Après avoir décrit les caractéristiques de l'école efficace, le rapport analyse comment les vingt-six projets soutenus par la Banque mondiale pour développer la qualité de l'enseignement primaire dans l'Afrique sub-saharienne prennent en compte ces critères.

14 MORRIS Paul. "Asia's Four Little Tigers: a Comparison of the Role of Education in their development ». Comparative Education, mars 1996, vol. 32, n 1, p. 95-109.

Cet article examine le rôle joué par l'éducation dans le développement rapide de Hongkong, Taiwan, la Corée du Sud et Singapour, qui présentent, dans ce domaine, des traits communs mais aussi d'importantes différences.

CONSEIL SUPÉRIEUR DE L'ÉDUCATION. Pour la réforme du système éducatif: dix années de consultation et de réflexion: avis au ministre de l'Education. Québec: Sainte-Foy: Conseil 
supérieur de l'éducation, 1995, 65 p.

Le Conseil a condensé dans ce document ses principaux diagnostics, propositions d'orientations et suggestions d'actions prioritaires formulés en dix ans pour améliorer le système éducatif. Il les a regroupés en trois parties: les fondements de toute amélioration ; les redressements à effectuer dans certains aspects du système ; les actions prioritaires pour chaque niveau ou secteur d'enseignement.

TEDGA Jean-Paul coord., et al. «Spécial Maroc ». Afrique éducation, mars 1996, n 19, p. 5-49.

Les différents enjeux éducatifs sont débattus par des spécialistes marocains. Les principaux points abordés sont : l'adéquation formation-emploi, la place de l'islam dans l'enseignement en tant que système de valeurs, les langues d'enseignement (arabe, français, berbère), la formation des maittres, le financement de l'éducation, la scolarisation des filles, l'organisation de l'enseignement supérieur et l'essor de l'enseignement supérieur privé, la coopération universitaire.

\section{Europe}

ASSOCIATION DES INSPECTEURS GÉNÉRAUX DE L'ÉDUCATION NATIONALE ET DE L'INSTRUCTION PUBLIQUE. Formation des élites et école pour tous : actes de la journée d'étude 1995. Paris : Ministère de l'Éducation nationale de l'Enseignement supérieur et de la Recherche, 1995, 213 p. Conférences, tables rondes et interventions de la journée du 13 septembre 1995 ont porté sur un problème inhérent aux systèmes éducatifs des sociétés développées : comment démocratiser l'enseignement et réduire l'échec scolaire tout en préservant la formation d'une élite. Les diverses contributions ont apporté des éclairages sur ce thème, dans des champs aussi variés que la physiologie, la neurobiologie, la sociologie, la psychologie, la créativité.

ASSOCIATION FRANÇAISE DES ADMINISTRATEURS DE L'ÉDUCATION. «L'école est-elle à l'heure de son temps?: actes du XVIII e colloque de l'AFAE, 29-30-31 mars 1996", Administration et éducation, $3^{e}$ trim. 1996, $n^{\circ}$ 71, p. 3-173.

Parfois tentée de se replier sur elle-même et de s'arracher au temps, l'école doit cependant réagir et s'adapter aux évolutions de la société. Si le tout-instruction n'est plus envisageable, l'insertion professionnelle seule ne peut, de son côté, constituer un objectif à long terme. Par ailleurs, la société demande à l'école d'être un lieu où se transmettent les savoirs, mais aussi où s'élaborent et s'affirment des valeurs et où se construit la notion de citoyenneté. La pluralité des réponses à l'interrogation initiale, à travers conférences, table ronde et ateliers, fait la richesse de ce colloque.

ASSOCIATION FRANÇAISE DES ADMINISTRATEURS DE L'ÉDUCATION. «L'école peut-elle, doit-elle préparer à un métier?: actes du XVII colloque de l'AFAE, 7-8-9 avril 1995 ", Administration et éducation, $3^{e}$ trim. 1995, $n^{\circ}$ 67, p. 3-172.

Communications et débats, dont ce numéro reprend le texte intégral, ont porté sur la formation et l'insertion professionnelles, les relations école-entreprise, la place et le rôle de l'établissement scolaire français dans ce contexte.

BENOÎT-GUILBOT Odile éd., et al. "Contre le chômage, la formation? Variations européennes ", Sociologie du travail, nov. 1995, vol. XXXVII, $n^{\circ} 4, p .495-755$.

L'importance accordée à la formation pour résoudre les problèmes du chômage est mise en cause dans ce numéro qui a fait appel à des sociologues mais aussi à des chercheurs en économie, économie du travail, sciences politiques. Si la formation n'est pas le remède 
miracle contre le chômage, quels sont alors les choix possibles en matière de politique éducative? Les articles permettent une comparaison européenne et une avancée du débat.

BRAJNIK Evguénia, FAURE Martine. "Les nouvelles tendances du système éducatif russe ». Revue internationale d'éducation - Sèvres, mars 1996, $n^{\circ}$ 9, p. 13-20.

L'évolution du système depuis la loi de 1992 sur l'éducation est présentée sous deux aspects : description des structures de l'enseignement ; analyse de la réforme en cours.

BYRAM Michaël, ZARATE Geneviève. Les jeunes confrontés à la différence : des propositions de formation. Strasbourg: Conseil de l'Europe, 1996, $66 \mathrm{p}$.

Contribution à la «Campagne européenne de la jeunesse contre le racisme, la xénophobie, l'antisémitisme et l'intolérance ", cette brochure, également publiée en anglais, a été réalisée dans le cadre du projet « Apprentissage des langues et citoyenneté européenne » du Conseil de l'Europe. Elle propose aux enseignants et éducateurs thèmes et activités pratiques pour faciliter la compréhension interculturelle. Après avoir clarifié les notions utilisées, elle développe divers thèmes : comportements envers les langues et dialectes, situation de gêne et malentendu culturel, reconnaissance des stéréotypes, analyse des médias.

CABAU Béatrice. "Suède: un vent de réformes". Revue internationale d'éducation - Sèvres, mars 1997, $n^{\circ} 13, p .11-20$.

Les récentes réformes de l'enseignement entraînent une nouvelle organisation du système éducatif, une décentralisation de la gestion, une expansion de l'enseignement libre, une transformation du mode d'évaluation.

CAMILLERI Carmel dir. Différence et cultures en Europe, Strasbourg: Conseil de l'Europe, 1995, $221 p$.

Ce document a été rédigé dans le cadre du projet «Démocratie, droits de l'homme et minorités : aspects éducatifs et culturels » élaboré par le Conseil de la coopération culturelle. Ses trois parties portent sur: l'altérité et les différences culturelles; les sociétés pluriculturelles et l'interculturalité ; le multiculturalisme et les institutions.

CERCLE DE RECHERCHE ET D'ACTION PÉDAGOGIQUES (Paris). "Demain l'école: manifeste ", Cahiers pédagogiques, sept. 1995, supplément, p. 1-24.

Ce document veut proposer, sur le système scolaire français, quelques analyses susceptibles de trouver un prolongement concret. Les thèmes abordés sont: les responsabilités et les pouvoirs de l'école, l'accès au patrimoine culturel et à la citoyenneté, la réponse aux besoins de la société, les moyens pédagogiques.

7 CERYCH Ladislav coord., et al. "Educational Reforms in Central and Eastern Europe ", European Journal of Education, mars 1996, vol. 31, n 1, p. 5-107.

L'Europe centrale et orientale connaît une période de transition qui a des répercussions sur l'enseignement, tant en ce qui concerne les structures que les programmes, la gestion, le financement, les enseignants. Cinq des articles, dont deux centrés sur l'enseignement secondaire, abordent ces thèmes, cependant que les deux derniers présentent la réforme de l'enseignement en Bulgarie et en Roumanie.

CHARLOT Bernard dir., BEILLEROT Jacky dir. La construction des politiques d'éducation et de formation: deuxième biennale de l'éducation et de la formation, Paris: PUF, 1995, 293 p., (Pédagogie d'aujourd'hui).

La sélection de textes présentée dans l'ouvrage a été établie suivant plusieurs axes : le politique et la planification ; le mondial, l'européen, le national et le local ; le scolaire et le 
non-scolaire. L'ouvrage comprend cinq parties: perspectives internationales; planification de l'éducation; exemples de recherches sur les systèmes scolaires et universitaires; rapports entre l'État et le «local » dans les politiques d'éducation et de formation ; les politiques de formation des entreprises. apprendre - vers la société cognitive. Luxembourg: Office des publications officielles des Communautés européennes, $1995,107 \mathrm{p}$.

Après une analyse des changements provoqués par la mondialisation des échanges, l'avènement de la société de l'information et l'accélération de la révolution scientifique et technique, le document propose, pour y répondre, de revaloriser la culture générale et de développer l'aptitude à l'emploi. Il suggère cinq objectifs d'action; encourager l'acquisition de connaissances nouvelles; rapprocher l'école et l'entreprise en développant l'apprentissage ; lutter contre l'exclusion en ouvrant une deuxième chance par l'école; maitriser trois langues communautaires; traiter sur un plan égal l'investissement physique et l'investissement en formation. Une version anglaise a été publiée en 1996.

COMMISSION EUROPÉENNE. Groupe de réflexion sur l'éducation et la formation, REIFFERS JeanLouis, rapport Accomplir l'Europe par l'éducation et la formation, Luxembourg: Office des publications officielles des Communautés européennes, 1997, $161 \mathrm{p}$.

Ce rapport résulte des travaux du groupe mis en place par la Commission en juillet 1995, pour approfondir les pistes ouvertes par le Livre blanc, et constitué de vingt-cinq experts. Plusieurs grands axes se dégagent de leurs travaux : construire la citoyenneté européenne et maintenir la cohésion sociale par l'éducation et la formation, préserver l'emploi et renforcer la compétitivité européenne, utiliser pleinement les ressources offertes par les technologies de l'information, enfin, dynamiser les systèmes d'éducation et de formation.

Examens des politiques nationales d'éducation : France. Paris : OCDE, 1996, $272 p$.

La notion d'égalité des chances constitue le fil conducteur de cette analyse. La première partie reprend le rapport de base préparé par le ministère de l'Éducation nationale. La seconde, élaborée par les examinateurs de l'OCDE, examine trois grandes innovations : les zones d'éducation prioritaires, la réforme de la formation des maîtres, les nouveaux diplômes « intermédiaires » dans l'enseignement technique et professionnel, et fait des suggestions pour renforcer le potentiel d'innovation et de pilotage stratégique du système éducatif.

FIALAIRE Jacques. L'école en Europe, Paris: Documentation française, 1996, 208 p., (Vivre en Europe).

Envisageant surtout la réalité institutionnelle de l'école, l'étude présente l'enseignement maternel, primaire et secondaire dans l'Europe des Quinze, en faisant ressortir les préoccupations communes aux différents pays. La première partie décrit les institutions politiques et administratives sur lesquelles reposent les systèmes éducatifs, avec, sousjacents, les grands principes tels que le droit à l'éducation, la laïcité, la loi, la norme scolaire. La deuxième partie fait une étude plus concrète du travail et de la vie scolaire et la troisième porte sur la gestion des établissements et les coûts de l'enseignement. Le livre se clôt sur la dimension européenne et la place prise par les actions européennes dans le domaine de l'éducation. 
du Luxembourg, Paris, Paris : Hachette Éducation, 1996, 175 p.

Ce colloque, qui avait pour thème l'évolution de la notion de territoire dans ses liens avec l'école, a pris en compte trois axes de réflexion: place et mission de l'école dans une perspective historique et géographique ; dimension de repère, d'intégration et de culture de l'institution scolaire; attentes auxquelles elle doit répondre pour mieux contribuer à la formation et au développement de tous.

LASONEN Johanna ed. Reforming Upper Secondary Education in Europe: Surveys of Stratégies for post-16 Education to Improve the Parity of Esteem for Initial Vocational Education in Eight European Educational Systems. Jyvdskyld (Finlande): University. Institute for Educational Research, 1996, XXXVI-295 p., (Publication. Séries B : Theory into practice, 92).

Cet ouvrage est un rapport d'étape sur le projet « Post-16 Stratégies » mis en œuvre en 1996 pour une durée de deux ans, dans le cadre du programme Léonard de Vinci, en Autriche, Angleterre, Finlande, France, Allemagne, Norvège, Écosse, Suède. Ce projet ayant pour but de revaloriser l'enseignement professionnel en supprimant l'écart existant entre celui-ci et l'enseignement secondaire général, le rapport présente le statut de l'enseignement professionnel et les réformes éventuelles mises en place dans chaque pays. La dernière partie analyse les stratégies qui se dégagent de cette étude, afin d'aboutir à des solutions.

LEWIS Mary rapp. Intégrer les services pour les enfants à risque : Danemark, France, PaysBas, Royaume-Uni (Angleterre et Pays de Galles) et Suède, Paris : OCDE-CERI, 1996, 102 p.

Après avoir défini les notions en question, le rapport examine, pour chaque pays, l'action des pouvoirs publics, les recherches universitaires, les initiatives pratiques. Il présente des points de vue d'administrateurs et d'hommes de terrain, repère les avantages et inconvénients des mesures prises pour coordonner les services et en souligne les implications.

PAYET Jean-Paul coord., VAN ZANTEN Agnès coord., et al. «L'école et la question de l'immigration ", Revue française de pédagogie, oct-nov-déc. 1996, n 117, p. 5-149.

Ce numéro présente, à travers des textes issus de recherches récentes, des approches et des perspectives multiples : statistiques, ethnographiques, psychologiques, sociologiques, politiques. Par ailleurs, tout en faisant le point sur l'état de la recherche en France, il s'intéresse aux travaux britanniques et américains. Parcours et performances scolaires, question de l'intégration, construction identitaire, débat sur la laïcité, politiques éducatives, on peut regrouper sous ces thèmes les différents articles et notes de synthèse.

REVERCHON Antoine, et al. "Peut-on (encore) réformer l'école?". Le Monde de l'éducation, sept. 1995, $n^{\circ} 229$, p. 20-40.

Si les idées des pédagogues et les propositions des politiques pour changer l'enseignement ne manquent pas, leur mise en œuvre se fait difficilement. À travers des reportages sur le terrain et des interviews de penseurs et de responsables, ce dossier présente, en France, des réalisations novatrices mais aussi les freins au changement.

VANISCOTTE Francine. Les écoles de l'Europe : systèmes éducatifs et dimension européenne, Toulouse : IUFM, Paris : INRP, 1997, 351 p., (Horizons pour la formation).

La première partie de l'ouvrage apporte une information de base sur les systèmes éducatifs des quinze pays membres de l'Union européenne. Dans la seconde, l'auteur souhaite faire découvrir la «valeur ajoutée» que représente, pour l'éducation, la dimension européenne. Cette notion pourrait permettre d'avancer sur certaines questions essentielles qui touchent tous les pays, comme les élèves en difficulté ou la 
formation initiale et continue des enseignants. La coopération européenne en matière d'éducation a d'ailleurs évolué, comme le montrent l'historique des actions et les orientations actuelles.

39 VULLIAMY Graham, WEBB Rosemary. "Education during Political Transition in Poland", International Journal of Educational Development, avril 1996, vol. 16, $n^{\circ} 2, p .111-123$.

L'article présente une analyse comparative de l'éducation dans la Pologne communiste et postcommuniste. Certaines des modifications observées résultent des changements idéologiques (enseignement de l'histoire, éducation religieuse), d'autres, des transformations économiques.

INDEX

Mots-clés : autonomie, décentralisation, enseignant, finalité de l'éducation, formation des enseignants, politique éducative, qualité de l'enseignement, réforme de l'enseignement, système éducatif

Index géographique : Afrique, Amérique, Asie, Europe 